\title{
Synthesis and biological activity of oxadiazole and triazolothiadiazole derivatives as tyrosinase inhibitors.
}

\begin{abstract}
A series of 16 oxadiazole and triazolothiadiazole derivatives were designed, synthesized and evaluated as mushroom tyrosinase inhibitors. Five derivatives were found to display high inhibition on the tyrosinase activity ranging from 0.87 to $1.49 \mathrm{lM}$. Compound 5 exhibited highest tyrosinase inhibitory activity with an IC50 value of $0.87 \pm 0.16 \mathrm{lM}$. The in silico protein-ligand docking using AUTODOCK 4.1 was successfully performed on compound 5 with significant binding energy value of $5.58 \mathrm{kcal} / \mathrm{mol}$. The docking results also showed that the tyrosinase inhibition might be due to the metal chelating effect by the presence of thione functionality in compounds 1-5. Further studies revealed that the presence of hydrophobic group such as cycloamine derivatives played a major role in the inhibition. Piperazine moiety in compound 5 appeared to be involved in an extensive hydrophobic contact and a $2.9 \AA$ hydrogen bonding with residue Glu 182 in the active site.
\end{abstract}

Keyword: Oxadiazole; Triazolothiadiazole; Piperazine; Mushroom tyrosinase inhibitor. 